\title{
Evaluation of the scintillation loss for optical communication systems with direct detection
}

\author{
Nicolas Perlot \\ German Aerospace Center (DLR) \\ Institute of Communications and Navigation \\ Oberpfaffenhofen, P.O. Box 1116 \\ 82234 Wessling, Germany \\ E-mail: nicolas.perlot@dlr.de
}

\begin{abstract}
In optical communications through the atmosphere, the evaluation of a link feasibility often requires the quantification of the scintillation penalty in terms of power loss. To find how much additional optical power is needed to reach the bit-error-rate (BER) requirements, the optical-power fluctuations must be characterized as well as the response of the receiver to those fluctuations. In the present analysis, the directdetected optical power is assumed to be either lognormal or gammagamma distributed. To account for the dynamics of the atmospheric channel, a distinction is made between short-term and long-term BERs. For a simple On-Off Keying (OOK) modulation, expressions of scintillation losses are given for different system requirements. Specifically, an upper bound is set to any of the three following quantities: the long-term $B E R$, the probability of having a too-high short-term BER, or the mean time during which the short-term BER is too high. Results show that, without any fade mitigation, losses under moderate scintillation are considerable. Finally, a simple code-word approach shows how scintillation losses can be reduced by channel coding. (๑) 2007 Society of Photo-Optical Instrumentation Engineers. [DOI: 10.1117/1.2436866]
\end{abstract}

Subject terms: free-space optics; scintillation loss; intensity modulation and direct detection (IM/DD) systems; short-term bit-error rate (BER); long-term BER; channel coding.

Paper 060079R received Jan. 30, 2006; revised manuscript received Jun. 19, 2006; accepted for publication Aug. 1, 2006; published online Feb. 7, 2007.

\section{Introduction}

Free-space optical communications suffer from drawbacks in the atmosphere. One of these drawbacks is scintillation. Scintillation refers to the random optical-power fluctuations caused by atmospheric turbulence.

In the assessment of a link budget, it is of great interest to quantify in terms of power loss the penalty caused by scintillation. This amounts to calculating how much additional power is needed to overcome scintillation effects and thus to reach the required performance. This additional power is referred to as the scintillation loss. To evaluate the scintillation loss, it is necessary to define the performance that must be reached. The system performance is usually evaluated in terms of bit error rate (BER) after the bitdecision process. Several authors have analyzed the system performance under turbulence effects by providing the mean BER, which corresponds to a BER evaluated over a long term (practically, over several minutes). ${ }^{1-3}$ This is one possibility among others. When evaluating the quality of the communication in a dynamic channel, one may also look at the BER over a shorter duration and set some requirements on it. The BER requirements may depend on the channel coding, on the synchronization system, or on the possible higher communication protocols.

For systems with intensity modulation and direct detection (IM/DD), the signal is proportional to the received optical power. To study the impact of optical power fluctuations on the BER of an IM/DD link, a receiver model

0091-3286/2007/\$25.00 @ 2007 SPIE including the noise sources is required in addition to a channel model. Power fluctuations can then be transposed into a power penalty for link budget calculations.

This paper provides an overview of the different possible derivations of scintillation losses. First, a methodology section enumerates the general assumptions that are made. After defining a short-term BER and a long-term BER that characterize the atmospheric optical channel, we express the scintillation loss to be determined. Based on the short-term and long-term BERs, several target performances are possible. Three types of losses are considered, with a section devoted to each type. These loss types correspond to setting an upper bound on the three following quantities: the long-term BER, the probability that the short-term BER exceeds a given value, and the mean time during which the short-term BER exceeds a given value. Finally, channel coding is considered with a distinction between fluctuations that are slow or fast compared to the span of a code word.

\section{Methodology}

\subsection{General Assumptions}

We consider the optically transmitted data as a chain of bits. We assume that the characteristics of the fluctuating received optical power are known. Assuming further that the beam wave was coherent at the transmitter (e.g., TEM $_{00}$ Gaussian beam), two different stationary stochastic processes for the received optical power are considered: a lognormal process and a gamma-gamma process. ${ }^{1}$ Note that 
the optical power may depart from these distributions when jitter or wander of the beam axis on the receiver becomes significant. $^{4,5}$

A lognormal variable normalized to its mean (i.e., of mean equal to one) is characterized by its variance, which in our case will be referred to as the power scintillation index $\sigma_{P}^{2}$. A gamma-gamma variable normalized to its mean is characterized by two parameters $\alpha$ and $\beta$ (refer to the appendix). We use hereafter the power scintillation index $\sigma_{P}^{2}$ and the parameters $\alpha$ and $\beta$ to quantify scintillation. Their values actually depend on many factors such as the link distance, the turbulence strength, the emitted wave, the wavelength, and the size of the receiving aperture. In the computed and displayed results, we restrict ourselves to the case $\sigma_{P}^{2}<1$, considering that a power scintillation index larger than one sets the communication link into a too low quality level. (This restriction does not hold, however, when channel coding is considered.)

\subsection{Short-Term and Long-Term BERs}

The scintillation time scale is highly dependent on the speed of the turbulence eddies crossing the beam. Typically on the order of $10 \mathrm{~ms}$, it is usually much larger than a bit duration. ${ }^{6,7}$ Thus, the level of optical power coding for a symbol (e.g., the symbol 1) can be viewed as constant over a large number of bits, and a BER for this large number of bits can be estimated. This BER calculated on a short term is conditioned on the level of the received optical power.

Let $P$ be the highest optical power coding for a symbol and subject to scintillation [e.g., for an On-Off Keying (OOK) modulation, $P$ will code for the symbol 1]. We define $g_{0}$ as the function giving the short-term BER noted $B E R_{S T}$ for a particular received power $P$ :

$B E R_{S T}=g_{0}(P)$.

The transformation $g_{0}$ depends on the intensity modulation, on the receiver noises, and on the decision threshold. The long-term BER is defined as the short-term BER averaged over the possible values of the received power $P$. With $f_{P}$ the probability density function of $P$, we thus have

$$
\begin{aligned}
B E R_{L T} & =\left\langle B E R_{S T}\right\rangle=\int_{0}^{\infty} B E R_{S T} f_{P}(p) \mathrm{d} p, \\
& =\int_{0}^{\infty} g_{0}(p) f_{P}(p) \mathrm{d} p
\end{aligned}
$$

where angular brackets denote ensemble averaging.

\subsection{Scintillation Loss $\ell_{s c}$}

In general, a rough estimation of the quality of an IM/DD communication link can easily be done. In Fig. 1, typical curves of the $g_{0}$ function and of the cumulative density function $(\mathrm{CDF}) F_{P}$ of $P$ are drawn. The quality of the link is determined by the overlap region of the curves $g_{0}$ and $F_{P}$. Two different distributions of the received power $P$ are shown in Fig. 1: a problematic distribution and the distribution of a compensated power.

However, for a given link, the value of a scintillation loss may vary greatly depending on its definition. Also depending on the definition of the scintillation loss, the diffi-

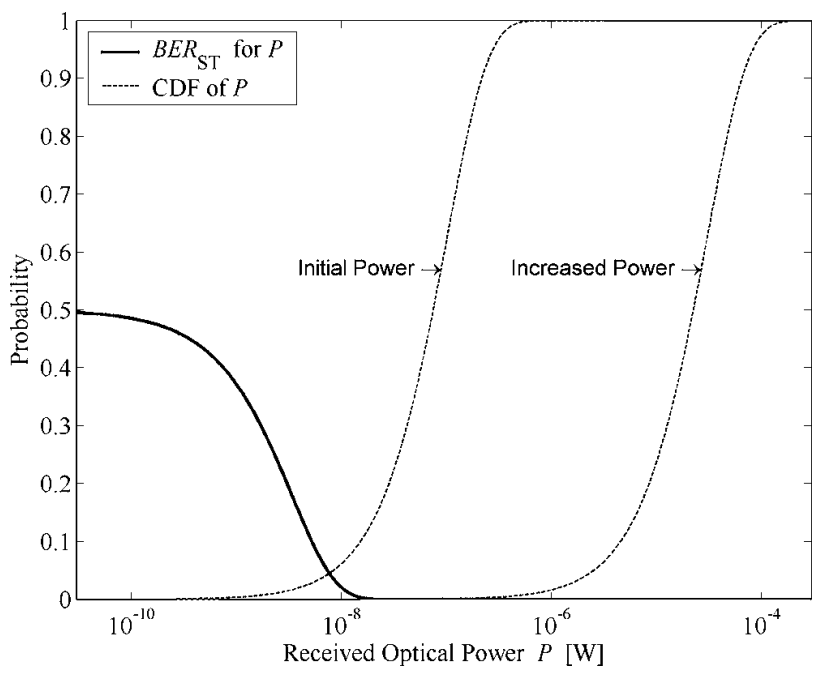

Fig. 1 Estimation of the link quality from the location of the curves $g_{0}(P)$ and $F_{P}(P)$. Increasing the transmitted power makes $F_{P}(P)$ shift to the right. Curves here are typical but arbitrary.

culty of its determination can vary greatly. In the expressions of scintillation loss (noted $\ell_{s c}$ ) provided in this paper, the performance to be reached depends on a reference BER, i.e., the BER on which the required performance is based. In Secs. 3-5, this reference BER, noted $B E R_{0}$, is chosen as the BER achieved without scintillation. It thus equals

$B E R_{0}=g_{0}(\langle P\rangle)$.

The loss $\ell_{s c}$ corresponds to the factor by which $P$ must be multiplied in order to fulfill the desired condition. The compensated signal is thus given by

$P_{\text {comp }}=\ell_{s c} P$.

\section{Loss Conditioned on the Long-Term BER}

In this first case, the transmitted signal must be compensated so that the long-term BER reaches the reference value $B E R_{0}$ :

$B E R_{L T}=B E R_{0}$.

This type of loss was considered in Ref. 8 with some restrictions, namely, a particular receiver model, a particular $B E R_{0}$, and the assumption of weak scintillation. Here we want to formulate this loss in a more general form. Using the definition of Eq. (2), we express the long-term BER of the compensated power as

$B E R_{L T}=\int_{0}^{\infty} g_{0}(p) f_{P, \text { comp }}(p) \mathrm{d} p$,

where $f_{P, \text { comp }}$ is the PDF of the compensated power $P_{\text {comp }}$. Using Eq. (4), Eq. (6) yields

$B E R_{L T}=\int_{0}^{\infty} g_{0}(p) \frac{1}{\ell_{s c}} f_{P}\left(\frac{p}{\ell_{s c}}\right) \mathrm{d} p$.

The condition formulated in Eq. (5) imposes 
$\int_{0}^{\infty} g_{0}(p) \frac{1}{\ell_{s c}} f_{P}\left(\frac{p}{\ell_{s c}}\right) \mathrm{d} p=g_{0}(\langle P\rangle)$.

We could have expected a scintillation loss to be independent of the amplitude of the transmitted power, i.e., independent of $\langle P\rangle$. But defining the scintillation loss by Eq. (8), this is not the case. To better see the dependence on the amplitude of the signal, we introduce the mean-normalized received optical power $P_{\text {norm }}$ :

$P_{\text {norm }}=\frac{P}{\langle P\rangle}$.

With $f_{P_{\text {norm }}}$ the PDF of $P_{\text {norm }}$, we can rewrite Eq. (8) as

$\int_{0}^{\infty} \frac{g_{0}(\langle P\rangle p)}{g_{0}(\langle P\rangle)} \frac{1}{\ell_{s c}} f_{P_{n o r m}}\left(\frac{p}{\ell_{s c}}\right) \mathrm{d} p=1$.

Therefore, the dependence of the loss $\ell_{s c}$ on $\langle P\rangle$ is given by the function $g_{0}$ and more precisely on the ratio $g_{0}(\langle P\rangle p) / g_{0}(\langle P\rangle)$. The loss $\ell_{s c}$ should then be found numerically.

Assuming the common OOK modulation, the equivalent of the $g_{0}$ function has been expressed in several publications (See, for example, Refs. 2, 3, and 8, and Sec. 7.5.1 of Ref. 1). The receiver noises are generally assumed to have a Gaussian distribution. Nevertheless, the receiver models defining $g_{0}$ differ in some additional assumptions that are made. Among these assumptions, the main differences consist of whether shot noise is negligible, whether the modulator extinction ratio is perfect, or whether the decision threshold is fixed or adaptive. Regarding the decision threshold, the value of a fixed threshold should minimize the long-term BER, whereas the values taken by an adaptive threshold should minimize the short-term BER at any time. The adaptive threshold, which offers better BER performance, can be implemented when bits are coded as a return-to-zero (RZ) signal or alternatively when the low frequencies including the slow turbulence-induced fluctuations are filtered from the received signal. Note, however, that there will be no large difference in the long-term BER whether one uses an optimal fixed threshold or an optimal adaptive threshold; this is because the long-term BER is mostly determined by high short-term BER values that a fixed threshold could minimize almost as well as an adaptive threshold.

$\ell_{s c}$ has been evaluated numerically assuming the OOK modulation and the following relatively simple expression for the $g_{0}$ function

$g_{0}(P)=Q\left[\frac{P / P_{0}}{1+\left(1+\xi_{0} P / P_{0}\right)^{1 / 2}}\right]$,

which was introduced in Ref. 8. In Eq. (11), $Q$ is the standard Gaussian tail integral defined by

$Q(x)=\frac{1}{\sqrt{2 \pi}} \int_{x}^{\infty} \exp \left[-\left(t^{2} / 2\right)\right] \mathrm{d} t$.

The characteristic power $P_{0}$ is related to the receiver floor noise (i.e., to the noise present in the receiver when no

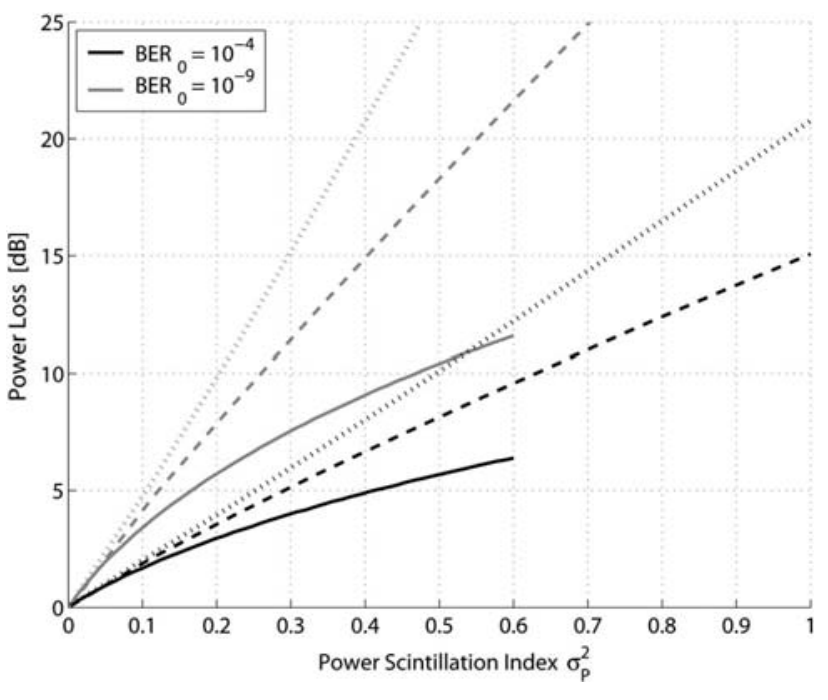

Fig. 2 Power loss $\ell_{s c}$ defined for a given long-term bit error rate $B E R_{0}$. Two different $B E R_{0}$ values and three different power distributions are considered: lognormal (solid line), gamma-gamma with $\alpha$ $=\beta$ (dashed line), and gamma-gamma with $\alpha=0.2 \beta$ (dotted line).

signal power is received), whereas the factor $\xi_{0}$ is related to the shot noise. To obtain Eq. (11), the optimal decision threshold is assumed adaptive and results from an approximation that forces the probability of missed detection and the probability of false alarm to be equal. (See Refs. 9 and 10 for more details on this approximation.) In addition, the modulator extinction ratio is assumed infinite. The parameters $P_{0}$ and $\xi_{0}$ of Eq. (11) have been adjusted so that $g_{0}$ fits the performance curve of the Fujitsu FRM5W621KT/LT Module consisting of an Avalanche photodiode, operating at a wavelength of $1550 \mathrm{~nm}$ and for a bit rate of $622 \mathrm{Mbit} / \mathrm{s}$. For this receiver, we found $P_{0}=1.35 \mathrm{nW}$ and $\xi_{0}=0.8$.

Figure 2 shows the losses conditioned on a long-term BER as a function of the power scintillation index. Because the lognormal distribution (solid line in the figure) is valid only under weak fluctuations where $\sigma_{P}^{2} \ll 1$, the corresponding losses are shown only up to a scintillation index of $\sigma_{P}^{2}=0.6$. We consider two types of gamma-gamma distributions: the case $\alpha=\beta$ (dashed line) and the case $\alpha=0.2 \beta$ (dotted line). According to the modified Rytov theory, the first case would be approximately obtained for the intensity of a spherical wave propagating through turbulence but remaining in the weak-fluctuation regime, ${ }^{1}$ whereas the case $\alpha=0.2 \beta$ would instead arise in the saturation regime. In the gamma-gamma distribution, $\alpha$ and $\beta$ have symmetric roles, and the scintillation index $\sigma_{P}^{2}$ is related to $\alpha$ and $\beta$ by

$\sigma_{P}^{2}=\frac{1}{\alpha}+\frac{1}{\beta}+\frac{1}{\alpha \beta}$

Looking at the receiver performance for a particular value of the power scintillation index $\sigma_{P}^{2}=0.5$, Fig. 3 shows the long-term BER as a function of the mean received power $\langle P\rangle$. In addition to the three distributions of Fig. 2, the performance curve without scintillation is plotted. The lognormal case at $\sigma_{P}^{2}=0.5$ may represent a near-ground link 


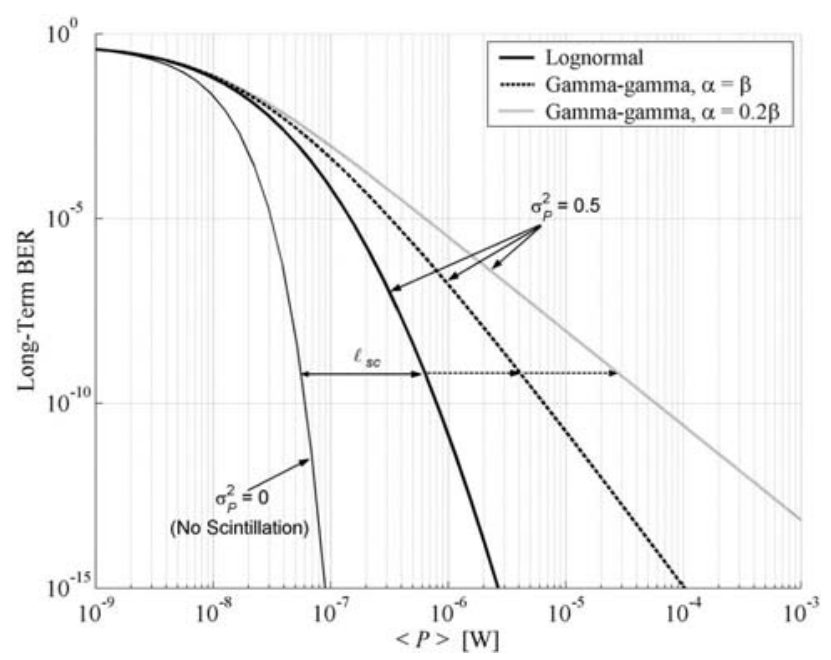

Fig. 3 Long-term BER with respect to the mean received power $\langle P\rangle$ under different scintillation conditions. Three different power distributions (the same as in Fig. 2) are considered with a scintillation index fixed at $\sigma_{P}^{2}=0.5$. A receiver model defined by Eq. (11) has been used. Losses can be viewed as the deviation of the obtained BER curves with respect to the "No Scintillation" curve.

of several hundreds of meters with a receiver having a point (i.e., non-extended) aperture. The gamma-gamma cases may describe longer links with significant aperture averaging at the receiver. ${ }^{1}$

\section{Loss Conditioned on a Maximum Probability for a Short-Term BER}

Here, the condition is to keep the probability of having a too-high short-term BER under a certain value. That is, we want to have, after compensation,

$\operatorname{Prob}\left(B E R_{S T}>B E R_{0}\right)=\theta_{0}$,

with $\theta_{0}$ the upper-bound probability. Equivalently, we write

$\operatorname{Prob}\left(P_{\text {comp }}<\langle P\rangle\right)=\theta_{0}$,

and

$\operatorname{Prob}\left(\frac{P}{\langle P\rangle}<\frac{1}{\ell_{s c}}\right)=\theta_{0}$.

Let $F_{P_{\text {norm }}}$ be the CDF of $P_{\text {norm }}$. Using the definition of $P_{\text {norm }}$ given by Eq. (9), Eq. (16) becomes

$F_{P_{\text {norm }}}\left(\frac{1}{\ell_{s c}}\right)=\theta_{0}$.

We finally find $\ell_{s c}$ as

$\ell_{s c}=\frac{1}{F_{P_{\text {norm }}}^{-1}\left(\theta_{0}\right)}$.

The scintillation loss $\ell_{s c}$ as expressed in Eq. (18) is thus independent of $\langle P\rangle$. A graphical interpretation of this type

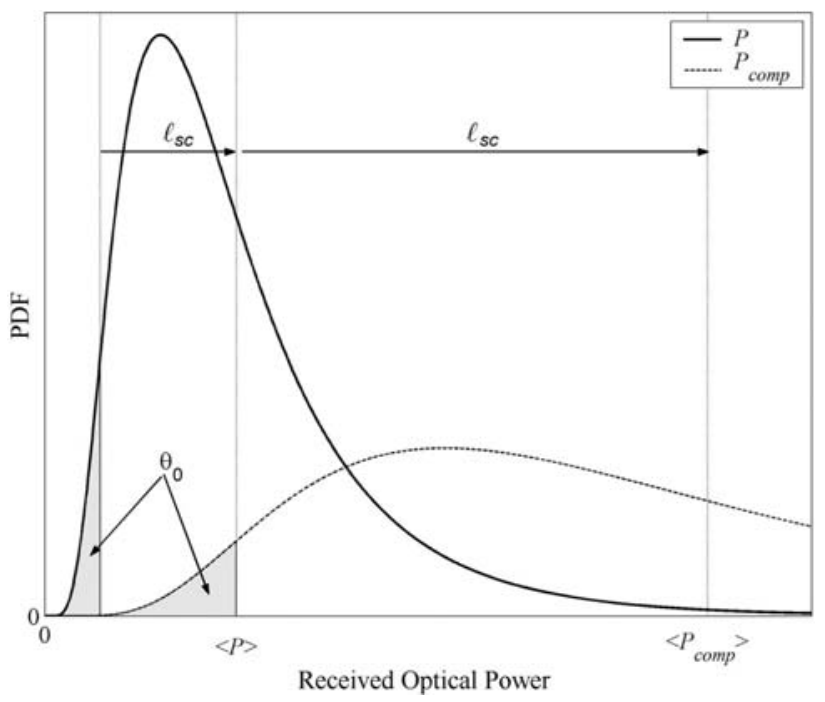

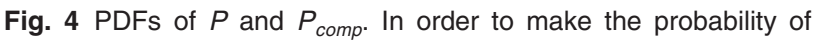
having a power less than $\langle P\rangle$ equal to $\theta_{0}$, the power must be compensated by a factor $\ell_{s c}$.

of loss can be seen in Fig. 4 with the PDFs of $P$ and $P_{\text {comp }}$. With a received optical power that is lognormally distributed, we have

$\ell_{s c}=\exp \left[-\operatorname{erfinv}\left(2 \theta_{0}-1\right) \sigma_{P} \sqrt{2}+\sigma_{P}^{2} / 2\right]$.

Equation (19) is an easy way to estimate the scintillation loss and was already used with $\theta=10^{-2}$ in the link budget of a successful transmission to a satellite. ${ }^{5,11}$ For a power that is gamma-gamma distributed, no tractable expression for the loss could be found. Figure 5 shows the computed power loss for $\theta_{0}=10^{-2}$ and $\theta_{0}=10^{-4}$.

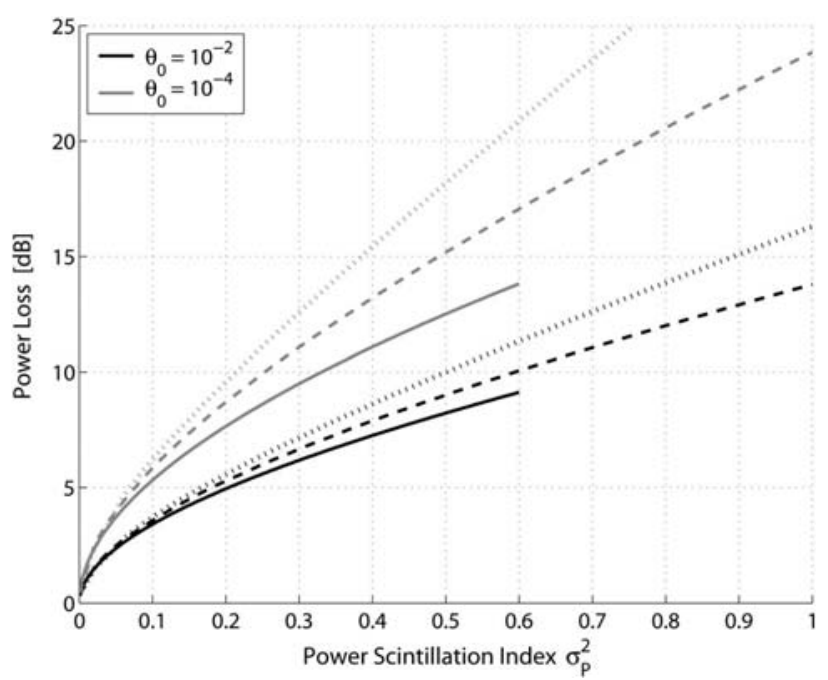

Fig. 5 Power loss $\ell_{s c}$ defined for a given probability $\theta_{0}$ that the short-term BER exceeds $B E R_{0}$. Two different $\theta_{0}$ values and three different power distributions are considered: lognormal (solid line), gamma-gamma with $\alpha=\beta$ (dashed line), and gamma-gamma with $\alpha=0.2 \beta$ (dotted line). 


\section{Loss Conditioned on a Maximum Mean Duration for a Short-Term BER}

The temporal fluctuations play an important role in the maintenance of a communication link and in the choice of a possible channel-coding scheme. We now want the mean time during which the short-term BER is higher than a given value $B E R_{0}$ to be lower than a given time $\tau_{0}$. So noting MeanTime $\left(B E R_{S T}>B E R_{0}\right)$, the mean time during which $B E R_{S T}$ is higher than $B E R_{0}$, the desired condition is:

$\operatorname{MeanTime}\left(B E R_{S T}>B E R_{0}\right)=\tau_{0}$,

which amounts to

$\operatorname{MeanTime}\left(P_{\text {comp }}<\langle P\rangle\right)=\tau_{0}$,

or

MeanTime $\left(\ell_{s c} P<\langle P\rangle\right)=\tau_{0}$.

We introduce the function $T_{P_{n o r m}}$, which gives the mean fade time for the normalized power $P_{\text {norm }}$, that is

$T_{P_{\text {norm }}}(p) \equiv \operatorname{MeanTime}\left(P_{\text {norm }}<p\right)$,

and then Eq. (22) becomes

$T_{P_{\text {norm }}}\left(\frac{1}{\ell_{s c}}\right)=\tau_{0}$

We finally find $\ell_{s c}$ as

$\ell_{s c}=\frac{1}{T_{P_{\text {norm }}}^{1}\left(\tau_{0}\right)}$.

The expression of the function $T_{P_{\text {norm }}}$ can be found from the relation ${ }^{12}$ :

$T_{P_{\text {norm }}}(p)=\frac{F_{P_{\text {norm }}}(p)}{\left\langle n_{P_{\text {norm }}}(p)\right\rangle}$,

where $F_{P_{\text {norm }}}$ is the CDF of $P_{\text {norm }}$ and where $\left\langle n_{P_{\text {norm }}}(p)\right\rangle$ is the expected number of fades per second that go below the threshold $p$. A graphical interpretation of this type of loss can be seen in Fig. 6. The loss as expressed by Eq. (25) is plotted in Fig. 7 for two different values (0.25 and 0.025) of the parameter $\gamma_{T}$ defined as the reduction factor of the mean time $\tau_{0}$ compared to the mean duration of a $0-\mathrm{dB}$ fade:

$\tau_{0}=\gamma_{T} T_{P_{\text {norm }}}(p=1), \quad$ with $\gamma_{T} \leqslant 1$.

$1 / T_{P_{\text {norm }}}(p=1)$ is close to the quasi-frequency $v_{0}$ of the power process (see the appendix) and is mostly determined by the atmospheric wind. We see in Fig. 7 that, for example, when the received power is lognormally distributed with a scintillation index of $\sigma_{P}^{2}=0.1$, the mean power must be increased by a factor of 10 to reduce the mean fade duration by a factor of 40 (i.e., with $\gamma_{T}=0.025$ ).

This type of loss may be helpful when designing a link that is supported by a channel-coding scheme. Indeed, one issue with the scintillation channel is that coding is ineffec-

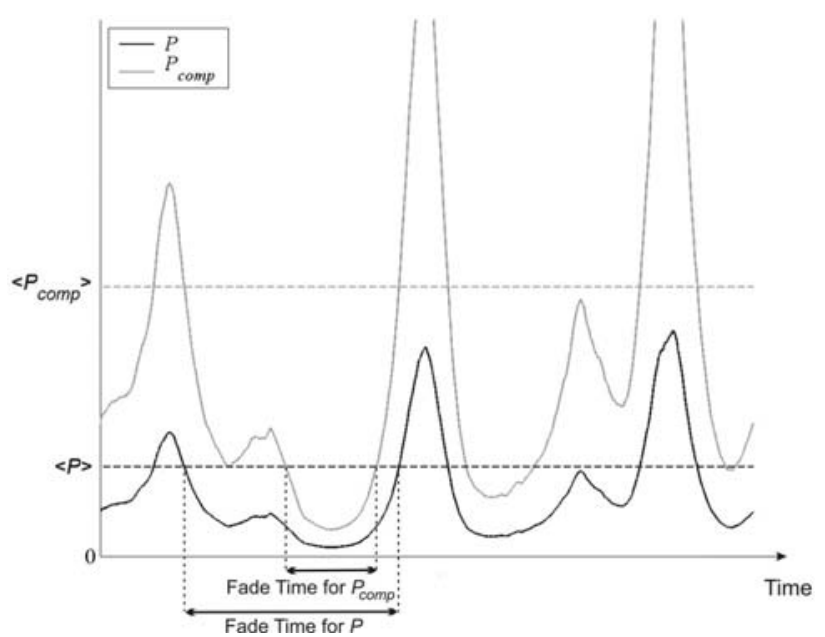

Fig. 6 Example of temporal realization for $P$ and $P_{\text {comp }}$ with fadetime reduction.

tive when a too-long series of bits is affected. ${ }^{7}$ Forwarderror-correction (FEC) codes are ineffective for deep fades of $10 \mathrm{~ms}$ when the bit rate is $1 \mathrm{Gbit} / \mathrm{s}$ or more. By increasing the level of transmitted power, we can attempt to reduce the mean fade duration and make the channel coding more effective. However, results displayed in Fig. 7 reveal that a power increase is an inefficient way of reducing the fade time and indicate that, for many scenarios, such a fade time reduction will not be strong enough to make a coding scheme more effective.

\section{Scintillation Loss with Channel Coding}

When channel coding is used to counteract scintillation, the requirements on the BER after the bit-decision stage are less restraining, and the scintillation loss can be greatly

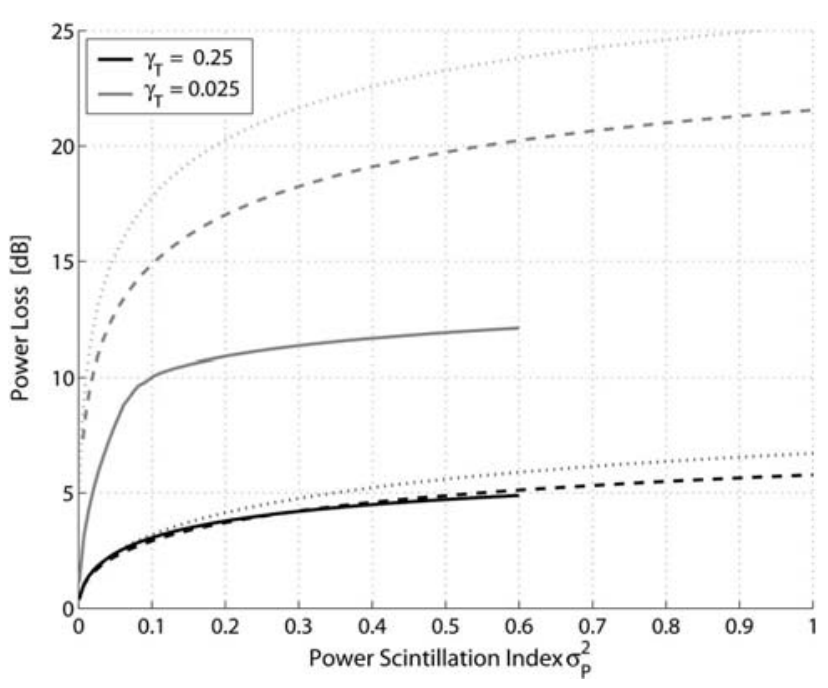

Fig. 7 Power loss $\ell_{s c}$ defined for a given mean time over which the short-term BER exceeds $B E R_{0}$. Two different $\gamma_{T}$ values and three different power distributions are considered: lognormal (solid line), gamma-gamma with $\alpha=\beta$ (dashed line), and gamma-gamma with $\alpha=0.2 \beta$ (dotted line). 
reduced or even suppressed. We consider a coding scheme that transforms a message of $k$ bits into a code word of $n$ bits with $n>k$. The redundancy $R$ of the code is defined by

$R=\frac{n-k}{n}$

The maximum number $n_{e, l i m}$ of correctable errors within this code word is given by ${ }^{13}$ :

$n_{e, l i m}=a(n-k)$,

where $a$ is a code-dependent factor that is less than 0.5 (with the value 0.5 being a theoretical limit). Inserting the code redundancy $R$ into Eq. (29), we obtain

$n_{e, l i m}=a R n$.

Thus, to correct all the errors within a code word, the number $n_{e}$ of bit errors within the word must satisfy:

$n_{e} \leqslant n_{e, \text { lim }}$.

Given the bit rate $B$ and the channel time constant $\tau_{\text {channel }}$, the typical number of bits over which the received power $P$ is constant is equal to $B \tau_{\text {channel }}$. For simplicity, we do not consider any interleaving, although the role of the interleaving/deinterleaving operation is merely to virtually reduce the channel time constant $\tau_{\text {channel }}$. Depending on the length $n$ of the code word with respect to $B \tau_{\text {channel }}$, we consider two cases where the number of errors $n_{e}$ can be related to either the short-term or the long-term BER according to

$n_{e}=\left\{\begin{array}{ll}n B E R_{S T}, & \text { if } n \ll B \tau_{\text {channel }} \\ n B E R_{L T}, & \text { if } n \gg B \tau_{\text {channel }} \gg 1\end{array}\right.$.

\subsection{Case $n \ll B \tau_{\text {channel }}$}

In this case, the fade is longer than a code word, and using Eq. (30), the condition of Eq. (31) becomes

$B E R_{S T} \leqslant a R$.

The short-term BER is a random variable, so Eq. (33) can be fulfilled only with a given probability. Let $\theta_{0}$ be the maximum allowed probability with which Eq. (33) is not fulfilled. We can then calculate a scintillation loss that is of the type described in Sec. 4 , and $\ell_{s c}$ is found by solving the following equation:

$\operatorname{Prob}\left[g_{0}\left(\ell_{s c} P\right)>a R\right]=\theta_{0}$.

\subsection{Case $n \gg B \tau_{\text {channel }} \gg 1$}

This case provides generally better coding performance but is rarely attained. ${ }^{7}$ The condition of Eq. (31) amounts here to

$B E R_{L T} \leqslant a R$.

If the transmit power needs to be compensated in order to have Eq. (35), the corresponding scintillation loss is of the

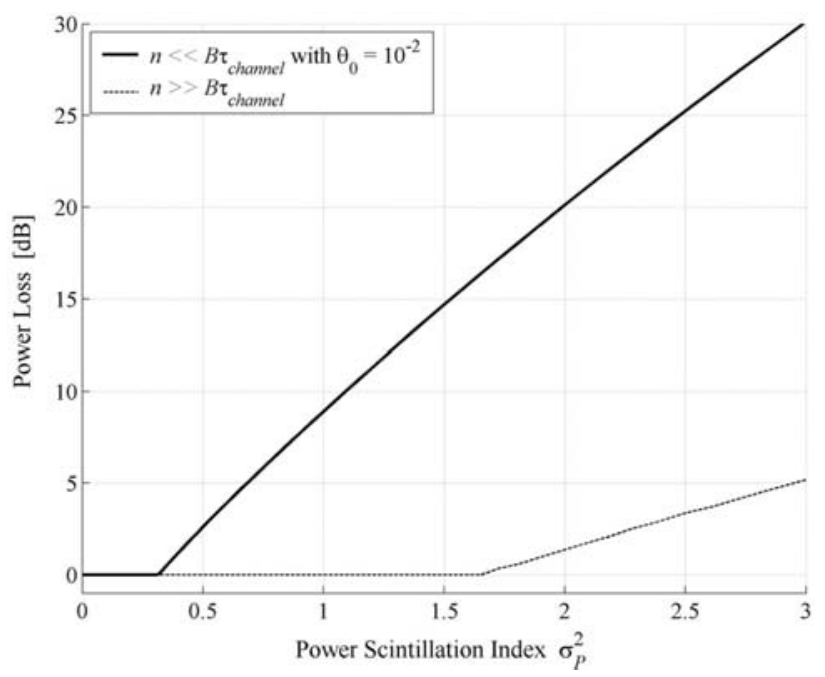

Fig. 8 Power loss $\ell_{s c}$ as a function of the scintillation index when a coding scheme is used with $a R=0.06$. The solid line corresponds to Eq. (34) with $\theta_{0}=10^{-2}$, whereas the dotted line corresponds to Eq. (36). The $g_{0}$ function is the same as in Sec. 3. The power is assumed gamma-gamma distributed with $\alpha=0.2 \beta$.

type described in Sec. 3, and $\ell_{s c}$ is found by solving the following equation:

$\left\langle g_{0}\left(\ell_{s c} P\right)\right\rangle=a R$.

To numerically apply Eqs. (34) and (36), we once more take $g_{0}$ as given by Eq. (11) with the same parameters $P_{0}$ and $\xi_{0}$ that fit the performance curve of the Fujitsu FRM5W621KT/LT Module at $B=622 \mathrm{Mbit} / \mathrm{s}$. We set $a R$ $=0.06$ (with, for example, $a=0.3$ and $R=0.2$ ), and we assume that the received power without scintillation gives a reference BER of $10^{-6}$, which leads to $\langle P\rangle=3.7 \times 10^{-8} \mathrm{~W}$. For the first case where fades are much longer than a code word, we set the maximum probability of correction failure to $\theta_{0}=10^{-2}$. Figure 8 shows the results for a gamma-gamma distribution with $\alpha=0.2 \beta$.

\section{Conclusion}

We have reviewed three different ways of determining in a link budget the power penalty associated with scintillation. No matter what type of loss is considered, losses are substantial for most practical scenarios $\left(\sigma_{P}^{2}>0.1\right)$ and for the common link requirements (e.g., $B E R_{L T}<10^{-6}$ ). The distribution of the received power affects greatly the scintillation loss. For an equal value of $\sigma_{P}^{2}$, the lognormal distribution leads to lower losses than the gamma-gamma distribution, and the worst case is obtained with the gamma-gamma distribution having either $\alpha$ or $\beta$ equal to zero (which amounts to a simple gamma distribution). This high sensitivity to the power distribution can be a problem in terms of result accuracy. Because of the complex nature of atmospheric turbulence, uncertainty in the predicted power PDF cannot be avoided (e.g., the choice between a lognormal and a gamma-gamma model at the beginning of the saturation regime may be equivocal). In turn, this PDF uncertainty 
can lead to a large uncertainty in the scintillation loss; this uncertainty can exceed $10 \mathrm{~dB}$ for large scintillation indices $\left(\sigma_{P}^{2}>0.5\right)$.

Certainly, strong fluctuations of the received power require the use of either techniques of scintillation mitigation (e.g., transmitter/receiver spatial diversity) or channel coding possibly combined with interleavers. The case of transmission with channel coding has been considered using simple relations from the theory of code words and providing altered expressions of the scintillation loss. These expressions predict a great reduction of the loss also under strong fluctuations, provided that fades are not too long.

\section{Appendix: Characterization of a Lognormal Process and a Gamma-Gamma Process}

\subsection{Lognormal Process}

The probability density function of a lognormal variable $P$ with small variance is

$$
\begin{aligned}
f_{P}(p)= & \frac{1}{p\left(2 \pi \sigma_{P}^{2}\right)^{1 / 2}} \exp \left\{-\frac{1}{2 \sigma_{P}^{2}}\left[\ln \left(\frac{p}{\langle P\rangle}\right)+\frac{1}{2} \sigma_{P}^{2}\right]^{2}\right\}, \\
& p>0,
\end{aligned}
$$

where $\sigma_{P}^{2}$ is the mean-normalized variance and where we used the approximation $\ln \left(\sigma_{P}^{2}+1\right) \approx \sigma_{P}^{2}$. It has been shown that, for lognormal processes, the mean number of fades below $p_{t h}$ per second takes the form ${ }^{14,15}$ :

$\left\langle n_{P}\left(p_{t h}\right)\right\rangle=v_{0} \exp \left\{-\frac{\left[\ln \left(\frac{p_{t h}}{\langle P\rangle}\right)+\frac{1}{2} \sigma_{P}^{2}\right]^{2}}{2 \sigma_{P}^{2}}\right\}$,

where

$v_{0}=\frac{1}{2 \pi}\left(-\frac{B_{P, \tau}^{\prime \prime}(0)}{B_{P, \tau}(0)}\right)^{1 / 2}$,

is the so-called quasi-frequency, with $B_{P, \tau}(0)$ and $B_{P, \tau}^{\prime \prime}(0)$ being respectively the temporal covariance function of the power $P$ and its second time derivative evaluated at the origin $\tau=0$. Note that to use Eq. (39), $B_{P, \tau}^{\prime \prime}(0)$ must exist.

\subsection{Gamma-Gamma Process}

The probability density function of a gamma-gamma variable $P$ is

$$
\begin{aligned}
f_{P}(p)= & \frac{2(\alpha \beta)^{(\alpha+\beta) / 2}}{\Gamma(\alpha) \Gamma(\beta)\langle P\rangle}\left(\frac{p}{\langle P\rangle}\right)^{(\alpha+\beta) / 2-1} K_{\alpha-\beta}\left[2\left(\frac{\alpha \beta p}{\langle P\rangle}\right)^{1 / 2}\right], \\
& p>0 .
\end{aligned}
$$

For gamma-gamma processes, Andrews et al. showed that the mean number of fades below $p_{\text {th }}$ per second is given by ${ }^{1}$ :

$$
\begin{aligned}
\left\langle n_{P}\left(p_{t h}\right)\right\rangle= & v_{0} \frac{2\left(2 \pi \sigma_{P}^{2} \alpha \beta\right)^{1 / 2}}{\Gamma(\alpha) \Gamma(\beta)}\left(\alpha \beta \frac{p_{t h}}{\langle P\rangle}\right)^{(\alpha+\beta-1) / 2} \\
& \times K_{\alpha-\beta}\left[2\left(\alpha \beta \frac{p_{t h}}{\langle P\rangle}\right)^{1 / 2}\right],
\end{aligned}
$$

where $v_{0}$ is given by Eq. (39).

\section{References}

1. L. C. Andrews, R. L. Phillips, and C. Y. Hopen, Laser Beam Scintillation with Applications, SPIE Press, Bellingham, WA (2001).

2. J. C. Ricklin, S. Bucaille, and F. M. Davidson, "Performance loss factors for optical communication through clear air turbulence," in Free-Space Laser Communication and Active Laser Illumination III, Proc. SPIE 5160, 1-12 (2004).

3. L. C. Andrews and R. L. Phillips, "Free space optical communication link and atmospheric effects: single aperture and arrays," in FreeSpace Laser Communication Technologies XVI, Proc. SPIE 5338B, 265-275 (2004).

4. K. Kiasaleh, "On the probability density function of signal intensity in free-space optical communications systems impaired by pointing jitter and turbulence," Opt. Eng., 33(11), 3748-3757 (1994).

5. M. Toyoshima, S. Yamakawa, T. Yamawaki, K. Arai, M. Reyes, A. Alonso, Z. Sodnik, and B. Demelenne, "Ground-to-satellite optical link tests between Japanese laser communications terminal and European geostationary satellite ARTEMIS," in Free-Space Laser Communication Technologies XVI, Proc. SPIE 5338A, 1-15 (2004).

6. K. Kiasaleh, "Performance analysis of free-space on-off-keying optical communication systems impaired by turbulence," in Free-Space Laser Communication Technologies XIV, Proc. SPIE 4635, 150-161 (2002).

7. H. Henniger, D. Giggenbach, F. David, and C. Rapp, "Evaluation of FEC for the atmospheric optical IM/DD channel," in Free-Space Laser Communication Technologies XV, Proc. SPIE 4975, 1-11 (2003).

8. F. David, "Scintillation loss in free-space optical IM/DD systems," in Free-Space Laser Communication Technologies XVI, Proc. SPIE 5338A, 65-75 (2004).

9. J. R. Barry and E. Lee, "Performance of coherent optical receivers," Proc. IEEE 78(8), 1369-1394 (1990).

10. S. B. Alexander, Optical Communication Receiver Design, SPIE Optical Engineering Press, Bellingham, WA (1997).

11. M. Toyoshima, S. Yamakawa, T. Yamawaki, K. Arai, M. Reyes, A. Alonso, Z. Sodnik, and B. Demelenne, "Long-term statistics of laser beam propagation in an optical ground-to-geostationary satellite communications link," IEEE Trans. Antennas Propag. 53(2), 842-850 (2005).

12. S. O. Rice, "Mathematical analysis of random noise," Bell Syst. Tech. J. 23, 282-332 (1944); 24, 46-156 (1945).

13. B. Friedrichs, Kanalcodierung-Grundlagen und Anwendungen in modernen Kommunikationssystemen, Springer, Berlin (1996).

14. H. T. Yura and W. G. McKinley, "Optical scintillation statistics for IR ground-to-space laser communication systems," Appl. Opt. 22, 33533358 (1983).

15. P. Beckman, Probability in Communication Engineering, Harcourt, Brace \& World, New York (1967).

Nicolas Perlot received his MS and his PhD in electronics engineering at the University of Valenciennes, France, in 2002 and 2005, respectively. He joined the Optical Communication Group of the German Aerospace Center (DLR) in Oberpfaffenhofen, Germany, in 2002. His research interests are atmospheric beam propagation, free-space optical communications, and characterization and simulation of random processes. 